\title{
A Case Report, Cerebral Salt Wasting Syndrome in An Adult
}

\author{
Charles Marihot Sibagariang ${ }^{\mathrm{a}}$, Soebagijo Poegoeh Edijanto \\ acharles.sibagariang@gmail.com \\ ${ }^{a}$ Resident of Clinical Pathology Department, Faculty of Medicine Airlangga University, Dr. Soetomo Hospital, Surabaya, Indonesia \\ ${ }^{b}$ Department of Clinical Pathology, Faculty of Medicine Airlangga University, Dr. Soetomo Hospital, Surabaya, Indonesia
}

\begin{abstract}
Background: Cerebral Salt Wasting Syndrome (CSW) is a state of sodium loss from the kidneys due to cerebral disorders that cause hyponatremia and decreased extracellular fluid volume. One study found that the main cause of hyponatremia was inappropriate electrolytic therapy, use of diuretics, and a Syndrome of Inappropriate Antidiuretic Hormone (SIADH) as much as $78 \%$, while CSW was as much as $10 \%$. CSW is a cause of hyponatremia which is very difficult to understand and challenging thus, it is very important to distinguish it from the SIADH. This is due to the different management between CSW and SIADH.

Case Description: A 47-year-old female complained of weakness, frequent thirst and urination, gradual loss of consciousness, and trembling hands. Physical examination revealed a pulse rate of $120 \mathrm{x} /$ minute. Laboratory results showed that serum Na was $108 \mathrm{mmol} / 1$, urine Na was $511.5 \mathrm{mmol} / 24 \mathrm{~h}$, urine production was $10.2 \mathrm{~L} / 24 \mathrm{~h}$, urine specific gravity $\leqq 1,005$, the fluid balance was minus $3.15 \mathrm{~L} / 24 \mathrm{~h}, \mathrm{AST} 177 \mathrm{U} / \mathrm{L}$, ALT 330 U/L, HbA1C $7.5 \%$, Random Blood Glucose $234 \mathrm{mg} / \mathrm{dL}$. MRI examination showed small vessel ischemia in the right and left semiovale centrum, periventricular and basal ganglia. Abdominal USG showed cholelithiasis.

Conclusion: Based on clinical symptoms and laboratory results especially hyponatremia, urine balanced, and MRI possibly the patient was diagnosed with CSW syndrome. Distinguishing CSW from most causes of hyponatremia is usually not difficult. This patient fulfills the diagnosis of Evans Syndrome, but an antiplatelet antibody test is still required. An accurate distinction between the two disorders is essential because SIADH is treated with fluid restriction, whereas CSW requires sodium and fluid replacement.
\end{abstract}

Key words: cerebral salt wasting, SIADH, hyponatremia.

\section{Introduction}

Hyponatremia is a state of electrolyte abnormalities when the serum sodium $\left(\mathrm{Na}^{+}\right)$concentration is $<136$ $\mathrm{mmol} / \mathrm{L}$ and most commonly encountered in clinical practice. Successful treatment of hyponatremia depends on an accurate diagnosis of the underlying etiology. ${ }^{1}$ The Syndrome of Inappropriate Antidiuretic Hormone (SIADH) is often diagnosed based on the state of hyponatremia, but it is important to distinguish it from Cerebral Salt Wasting Syndrome (CSW) which responds to very different treatments. ${ }^{2}$

CSW was first described by Peters et al. in 1950, as a clinical condition characterized by renal sodium loss leading to dehydration and hyponatremia in patients with intracranial neurologic disorders. ${ }^{3}$ Hyponatremia in patients with central nervous system disease was almost entirely ascribed to SIADH after Schwartz et al. identified SIADH in 1957. However, the finding that approximately $14 \%$ of SIADH patients have no abnormalities in ADH secretion suggests that mechanisms other than excessive $\mathrm{ADH}$ levels may lead to the clinical picture of SIADH. ${ }^{4} \mathrm{The}$ research of Cerdà et al. found that $14 \%$ of patients suffered from hyponatremia from 129 patients who were treated at the hospital's neurology unit. The main causes of hyponatremia were inappropriate electrolytic therapy, use of diuretics, and SIADH in $78 \%$ of patients, while CSW was in $10 \% .^{5}$

\section{Case presentation}

Female, 47 years old, referred from Al Irsyad Hospital with diagnosis hyponatremia and main complaint of feeling weak since 2 weeks before being admitted to the hospital. The patient also complains of easily falling asleep, hands moving uncontrolled, cough, fever. The patient complained of being thirsty, often woke up at night to urinate since 1 month before being admitted to the hospital. The general condition of the patient is weak, GCS 4-4-5, blood pressure 130/70 $\mathrm{mmHg}$, pulse $120 \mathrm{x} /$ minute, breathing $20 \mathrm{x} /$ minute, axillary temperature $38.00 \mathrm{C}$. Examination of the head did not reveal anemia, icterus, cyanosis and dyspnea. Thoracic examination revealed a symmetrical chest shape, without chest wall retraction. Single S1 S2 heart sound without gallops or heart murmurs. Breath sounds are vesicular, without crackles and wheezing. Abdominal examination, found bowel sounds normal, sociable, not palpable liver, spleen and kidney enlargement. Neurological examination revealed involuntary tremor.

Laboratory result: Hemoglobin 13,9 g/dL, WBC 5,89 x 103/ $\mu \mathrm{L}$, Platelets 264 x $10^{3} / \mu \mathrm{L}, \mathrm{BUN} 5 \mathrm{mg} / \mathrm{dL}$, Serum creatinine 0,47 mg/dL, Albumin 3,6 g/dL, AST 177 IU/L, ALT 330 IU/L, Random Blood Glucose 118 $\mathrm{mg} / \mathrm{dL}$, Cholesterol $144 \mathrm{mg} / \mathrm{dL}$, HDL $30 \mathrm{mg} / \mathrm{dL}$, LDL $95 \mathrm{mg} / \mathrm{dL}$. There is decrement of Natrium $108 \mathrm{mmol} / \mathrm{L}$, Kalium 2,8 mmol/L and Chloride $80 \mathrm{mmol} / \mathrm{L}$. We followed the urine test showed in table 1 and table 2. Chest $\mathrm{x}-$ IJRP 2021, 87(1), 23-26; doi:.10.47119/IJRP1008711020212363 
ray showed lung inflammation, Abdominal Ultrasound there is Cholelithiasis and from MRI found Small vessel ischemia in the centrum semiovale, periventricular and right and left basal ganglia.

Table 1. Urine test

\begin{tabular}{lccc}
\hline & $\mathbf{1 7 / 1 2 / 1 9}$ & $\mathbf{1 9 / 1 2 / 1 9}$ & Reference Range \\
\hline Potassium (mmol/24hr) & 63 & 36,8 & $\mathbf{3 5 - 8 0}$ \\
Sodium (mmol/24hr) & 209 & 511,5 & $\mathbf{3 0 - 3 0 0}$ \\
Chloride (mmol/24hr) & 274 & 517 & $\mathbf{8 5 - 1 7 0}$ \\
BUN & 12.160 & 7260 & \\
Creatinine (mg/24 hr) & 1.485 & 908 & $\mathbf{6 0 0 - 2 0 0 0}$ \\
osmolarity serum & 237,2 & &
\end{tabular}

Table 2. Urine output and balance

\begin{tabular}{|c|c|c|c|c|c|}
\hline & $\mathbf{1 8 / 1 2 / 1 9}$ & $\mathbf{1 9 / 1 2 / 1 9}$ & $\mathbf{2 0} / \mathbf{1 2} / \mathbf{1 9}$ & $\mathbf{0 2 / 0 1 / 2 0}$ & $\mathbf{0 3 / 0 1 / 2 0}$ \\
\hline $\begin{array}{c}\text { Urine Output } \\
\text { (Liter) }\end{array}$ & 6,7 & 8,2 & 10,2 & 15.3 & 12.1 \\
\hline $\begin{array}{c}\text { Urine Balance } \\
\text { (L/24hr) }\end{array}$ & $-3,6$ & $-3,15$ & $-4,5$ & & $-5,3$ \\
\hline
\end{tabular}

\section{Discussion}

CSWS is reported as a condition of increased urine volume and excessive sodium loss accompanied by weight loss in intracranial hemorrhagic disorders. Hyponatremia is a common complication of Central Nervous System disease, especially head injury and cerebrovascular disorders, and has been considered a generalized SIADH. ${ }^{6}$ Saleem et al., 2014 obtained approximately $67 \%$ SIADH and 33\% CSW in stroke patients with hyponatremia. ${ }^{7}$

The mechanisms underlying the association between CSW and severe neurologic disease have not been clearly identified. The maintenance of sodium and water homeostasis in the body is mostly arranged by the complicated interactions between the autonomic nervous system and humoral factors. Disruption of the normal interactions between these systems can result in a dysregulation of sodium and water at the nephron level, leading to more global changes in sodium and water. It has been postulated that impaired sympathetic impulses to the kidney and the presence of abnormally elevated circulating natriuretic factor may cause CSW. ${ }^{8}$

Yee AH et al., 2010 revealed that there are several postulates of CSW pathophysiology ${ }^{8}$ :

1. Sympathetic Nervous System Hypothesis

Peter's theory suggests that disturbances in the CNS affect salt and water balance mechanisms that could potentially impair the kidney's ability to maintain proper sodium homeostasis. Loss of adrenergic tone to the nephrons will have 2 important consequences : 1) decreased renin secretion by juxtaglomerular cells which will cause a decrease in aldosterone levels and sodium reabsorption, 2) cause dilation of afferent arterioles which increases plasma and sodium filtration in the glomerulus.

\section{Natriuretic Theory}

Natriuretic peptides are molecules that function to prevent excess water and salt retention by antagonizing the RAAS, causing vascular relaxation, and inhibiting sympathetic out-flow and formation of vasoconstrictor peptides. There are 4 natriuretic peptides associated with CSW, namely a) atrial natriuretic peptide (ANP)2; brain-natriuretic peptide (BNP)3; C-type natriuretic peptide (CNP); and 4 dendroaspis natriuretic peptide (DNP). Several hypotheses have been proposed to explain how intracranial disturbances may lead to elevated serum levels of this peptide. One plausible hypothesis is that direct damage to cortical and subcortical structures leads to the inadvertent release of hormones directly into the circulation. Myocardial tissue can also increase natriuretic peptide levels in CSW. Associated neurohumoral findings have also been demonstrated in other acute brain injuries, such as ischemic stroke. 


\section{Another hypothesis}

Kojima et al. found a significant increase in urine volume and sodium excretion, a decrease in body weight, and an increase in hematocrit in mice with CSW. ANP levels decreased, while BNP and ADH levels did not change. They concluded that an undefined mechanism, or one involving DNP, likely underlies the etiology of CSW

The difference between CSW and most causes of hyponatremia (diuretic use, adrenal insufficiency, extrarenal-induced volume depletion states, hypothyroidism, congestive heart failure) is usually not difficult. ${ }^{9}$ The challenge lies in the difference in CSW from SIADH, as it has similar serum and urine laboratory abnormalities and occurs in the same neurological disease and neurosurgery. ${ }^{10,11}$ An accurate distinction between the two disorders is very important (table 3), as misdiagnosis can lead to inappropriate therapy. SIADH is treated with fluid restriction, whereas CSW requires sodium and fluid replacement. ${ }^{10}$

Table 3. difference between CSW and SIADH ${ }^{8}$

\begin{tabular}{|c|c|c|}
\hline \multicolumn{3}{|c|}{ Differential diagnosis of CSW and SIADH } \\
\hline Variable & CSW & SIADH \\
\hline Urine osmolality & $\uparrow(>100 \mathrm{mOsm} / \mathrm{kg})$ & $\uparrow(>100 \mathrm{mOsm} / \mathrm{kg})$ \\
\hline Urine sodium concentration & $\uparrow(>40 \mathrm{mmol} / \mathrm{L})$ & $\uparrow(>40 \mathrm{mmol} / \mathrm{L})$ \\
\hline Extracellular fluid volume & $\downarrow$ & $\uparrow$ \\
\hline Body weight & $\downarrow$ & $\leftrightarrow$ or $\uparrow$ \\
\hline Fluid balance & Negative & Neutral to slightly + \\
\hline Urine volume & $\leftrightarrow$ or $\uparrow$ & $\leftrightarrow$ or $\downarrow$ \\
\hline Heart rate & $\leftrightarrow$ or $\uparrow$ & $\leftrightarrow$ \\
\hline Hematocrit & $\uparrow$ & $\leftrightarrow$ \\
\hline Albumin & $\uparrow$ & $\leftrightarrow$ \\
\hline Serum bicarbonate & $\uparrow$ & $\leftrightarrow$ or $\downarrow$ \\
\hline Blood urea nitrogen & $\uparrow$ & $\leftrightarrow$ or $\downarrow$ \\
\hline Serum uric acid & $\leftrightarrow$ or $\downarrow$ & $\downarrow$ \\
\hline Sodium balance & Negative & Neutral or + \\
\hline Central venous pressure & $\downarrow\left(<6 \mathrm{~cm} \mathrm{H}_{2} \mathrm{O}\right)$ & $\leftrightarrow$ or slightly $+\left(6-10 \mathrm{~cm} \mathrm{H}_{2} \mathrm{O}\right)$ \\
\hline Wedge pressure & $\downarrow$ & $\leftrightarrow$ or slightly $\uparrow$ \\
\hline
\end{tabular}

CSW is a syndrome of hypovolemic hyponatremia. The main clinical features are hypovolemia, often with negative fluid balance, serum hyponatremia and hypoosmolality, high urine osmolality, and high urine sodium. CSW differs from SIADH due to excessive renal excretion of sodium and water in CSW. ${ }^{8}$ In this case, there was hypovolemia with negative fluid balance, hyponatremia, serum hypoosmolarity, and also increased urine sodium levels with polyuria leading to CSW. CSW and SIADH are not possible to differentiate on the basis of serum and urine laboratory findings alone, because the associated abnormalities are identical. ${ }^{8}$ Accurate determination of the patient's volume status is the key to differentiating these syndromes. Unfortunately, the determination of volume status is very difficult to do accurately in routine clinical practice.

Liamis $\mathrm{G}$ et al.,2014 reported that DM patients have an association with the occurrence of hyponatremia, but the cause of hyponatremia is due to dilution, osmotic diuresis and other drugs. ${ }^{12}$ In this case, high HbA1C and GDA values were obtained, but there was no history of previous DM. The patient did not have a history of using drugs that can cause diuresis, so that DM caused hyponatremia to be ruled out. The increase in transaminase values in this patient was due to the presence of cholelithiasis based on abdominal ultrasound results. The authors found no literature that suggested an association between elevated transaminases and the CSW syndrome. CSW often occurs after brain injury or trauma, subarachnoid hemorrhage, brain surgery or stroke. Saleem et al., 2014 found that $35 \%$ of patients with hyponatremia in stroke had CSW syndrome. In this case, the possibility of CSW was due to an ischemic stroke that was identified based on the patient's MRI.

\section{Conclusion}

Based on clinical symptoms and laboratory results especially hyponatremia, urine balanced and MRI possibly the patient was diagnosed as CSW syndrome. Distinguishing CSW from most causes of hyponatremia is usually not difficult. This patient fulfills the diagnosis of Evans Syndrome, but an antiplatelet antibody test is still required. An accurate distinction between the two disorders is essential because SIADH is treated with fluid restriction, whereas CSW requires sodium and fluid replacement. 


\section{Acknowledgement}

The author would like to thank Mr. Prof. Soebagijo Poegoeh Edijanto, M.D., Ph.D., who has helped and guided the writing of these case report.

\section{References}

1. Chris Thompson, Tomas Berl, Alberto T GJ. Best Practice \& Research Clinical Endocrinology \& Metabolism Hypoparathyroidism. Clin Endocrinol Metab. 2012;26:517-22.

2. M J Brookes. in meningoencephalitis : a case Moderate hypoglycaemia obliterates working memory in. Br Med J. 2003;74:277-82.

3. Harshal Dholke, Ann Campos, C Naresh K. Reddy MKP. Cerebral salt wasting syndrome. Neuroanaesth Crit Care. $2016 ; 3^{3}: 205-10$.

4. Harrigan MR. Cerebral salt wasting syndrome. Neurosurg Rev. 1996;38 $: 152-8$.

5. Cerdà-Esteve M, Cuadrado-Godia E, Chillaron JJ, Pont-Sunyer C, Cucurella G, Fernández M, et al. Cerebral salt wasting syndrome: Review. Eur J Intern Med. 2008;194:249-54.

6. Tanaka T, Uno H, Miyashita K, Nagatsuka K. Cerebral salt-wasting syndrome due to hemorrhagic brain infarction: A case report. J Med Case Rep. 2014; $8^{1}: 1-5$.

7. Saleem S, Yousuf I, Gul A, Gupta S, Verma S. Hyponatremia in stroke. Ann Indian Acad Neurol. 2014; $17^{1}: 55-7$.

8. Yee AH, Burns JD, Wijdicks EFM. Cerebral Salt Wasting: Pathophysiology, Diagnosis, and Treatment. Neurosurg Clin N Am [Internet]. 2010;212:339-52. Available from: http://dx.doi.org/10.1016/j.nec.2009.10.011

9. $\quad$ Adrogue HJ MN. Hyponatremia. N Engl J Med. 2000;342:1581-1589.

10. Janicic N, Verbalis JG. Evaluation and management of hypo-osmolality in hospitalized patients. Endocrinol Metab Clin North Am. 2003;32²:459-81.

11. Smith DM, Mckenna K, Thompson CJ. Hyponatraemia. 2000;667-78.

12. Liamis G. Diabetes mellitus and electrolyte disorders. World J Clin Cases. $2014 ; 2^{10}: 488$. 